\title{
The Synchronization of the Human Cortical Working Memory Network
}

\author{
Sharlene D. Newman, Marcel Adam J ust, and Patricia A. Carpenter \\ Center for Cognitive Brain Imaging, Department of Psychology, Carnegie Mellon University, Pittsburgh, Pennsylvania 15213
}

Received April 10, 2001

\begin{abstract}
A verbal reasoning problem at the intersection of verbal working memory, problem-solving, and language comprehension was examined using event-related fMRI to distinguish differences in the differential timing of the response of the various cortical regions that compose the working memory network. Problems were developed such that the process demand as well as the timing of the manipulation of the contents of working memory (i.e., a demanding computation) was varied. Activation was observed in several regions including the dorsolateral prefrontal cortex, the inferior frontal gyrus, and the parietal lobe. Examination of the MR amplitude response revealed that the regions do not all activate simultaneously; instead, their activation time courses reveal differential responses that correspond to their theoretical processing role in the problem-solving task. The coordination of cortical area responses reveals how the various cortical regions synchronize and collaborate in order to accomplish a given cognitive function. $\odot 2002$ Elsevier Science (USA)
\end{abstract}

Key Words: fMRI; working memory; problem-solving.

\section{INTRODUCTION}

All forms of immediate thought, from reasoning to sentence comprehension, entail the manipulation and temporary storage of information, and for that reason, a central role is attributed to working memory. Working memory entails not only the systems that support the temporary storage of information, but it can also be viewed as the pool of operational resources that perform computations on that information. The analysis of the working-memory system has been a central focus of both behavioral and neuroimaging research. From this rich literature has emerged what seems to be highly integrated but separable working memory systems.

One such system is the phonological loop based on a model proposed by Baddeley (1986). The phonological loop is subserved by two functional units: a rehearsal system and a short-term buffer. Several neuroimaging studies have converged upon the cortical regions thought to be the basis of the phonological loop, the inferior frontal and inferior parietal regions (Awh et al., 1996; J onides et al., 1998). For example, a set of neuroimaging studies that examined verbal storage and rehearsal compared an item recognition task, an n-back working memory task, and two dual-tasks (one with a memory task and repetition and one with a memory task and finger tapping) (Awh et al., 1996). The prefrontal activation, concentrated in IFG, was activated to a greater extent for rehearsal and the posterior inferior parietal region was activated to a greater extent for storage.

The second of the working memory systems is composed of the dorsolateral prefrontal cortex and the region in and around the intraparietal sulcus. These regions have been found to be involved in several working memory tasks including both spatial (Rowe et al., 2000, 2001; Owen et al., 1999) and nonspatial tasks (D’E sposito et al., 1999). While these regions have been found to be involved in the maintenance of information, they seem to be especially involved when information being held on-line must be manipulated. For example, during a mental rotation task, activation was observed in both the DLPFC and the IPS (Carpenter et al., 1999). In addition the functional connectivity between the two regions increased with task difficulty (Diwadkar et al., 2000).

Event-related $\mathrm{FMRI}$ can provide a measure of the time course of brain activation as a function of the type of computational demand that is imposed. This new capability of neuroimaging allows us to examine not only which cortical regions become activated, but also how different cortical regions synchronize their activity to accomplish a computational task. The current event-related fMRI study examines verbal working memory in the context of solving problems that were patterned after the following prototype, which has proven to be enigmatically difficult (Casey, 1993):

\footnotetext{
"Imagine that a man is looking at a photograph while saying, 'Brothers and sisters have I none. That man's father is my father's son. Who is in the photograph?"
}

The solution is difficult. In a study with 101 adult participants, $77 \%$ of the respondents chose the same incorrect answer (the man himself), while only $13 \%$ chose the correct answer (it is a photograph of the 
man's son). Casey suggested that the various processing demands of this riddle may exceed verbal workingmemory capacity.

One such demand arises from the order of the two phrases That man's father and my father's son. Therefore, in the current event-related fMRI study we developed two versions of the "brothers" riddle that varied the order of the two noun phrases to distinguish differences in the timing of cortical responses that subserve part of the working memory network. In the first problem type (early/low load), comprehending the first half of the critical sentence requires an extra computation (computing the referent of "the first month after April"), whereas no such corresponding computation is required in the second part of the sentence. By contrast, in the second problem type (late/high load), the reverse is true.

\section{Early/Low load}

The first month after April is the month before my

favorite month. J une, J uly, Other

The structure of these two types of problems leads to some interesting contrastive predictions about the time course of the brain activation underlying the comprehension and solution. We predicted that there would be at least two different time courses of activation from various regions within the working memory network. First, cortical regions subserving the extra computation described above (determining the referent of the entity that is referred to indirectly, such as the month after April) should have a time course that re flects the timing of that computation (i.e., whether it occurs early or late in the problem). Therefore, in an earlier period, such regions should show a larger amplitude response for the early/low load condition compared to the late/high load condition and in a later period, these same regions should show a larger amplitude response for the late/high load condition compared to the early/low load condition, as shown in the upper panel of Fig. 1. One such region that might be expected to reveal such a response is the intraparietal sulcal region (Dehaene et al., 1999).

A second set of cortical regions is predicted to reveal a differential response between the two conditions only in the later period, due to the increased working memory load that results from the ordering of the two phrases in the late/high load condition. This extra working memory load arises in the late/high load problems because the definite reference of the first phrase (e.g., The day before my favorite day) cannot be computed until the second phrase has been read. The contents of the first phrase must be revisited (mentally or visually) after the referent of the second phrase has been determined and only then can the referent of the first phrase be computed. The buffering and delayed computation should produce activation in regions associated with verbal working memory and verbal processing. Such regions should reveal a greater signal amplitude response in the later period for the late/high load condition compared with the early/low load condition, as shown in the lower panel of Fig. 1. Due to the role of the dorsolateral prefrontal cortex in working memory processing, it is one of the regions that might be expected to reveal such a response (D'E sposito et al., 1999; Petrides, 1998).

\section{METHOD}

Participants. Fourteen neurologically normal partici pants from the Carnegie Mellon University community completed the current task during a fMRI session. All of the participants gave informed consent that was approved by the University of Pittsburgh and Carnegie Mellon Institutional Review Boards.

Experimental paradigm. The experiment consisted of two types of verbal problems (early/low load vs. late/high load), as described above. Three additional variables were manipulated, to introduce superficial variation among the problems: (1) distance from the reference point (e.g., the first, second, or third month after); (2) direction from reference point (i.e., before or after), and (3) problem domain (e.g., days, months, or letters).

The sentences were projected onto a transparent screen that was suspended from the upper surface of the scanner bore. The first half of the sentence (the top line in the examples) was presented alone on the screen for $4.5 \mathrm{~s}$. Afterwards the second half of the sentence was presented alone for $4.5 \mathrm{~s}$. The probe, which consisted of two possible targets and "Other," was then presented alone on the screen for $3 \mathrm{~s}$.

The experiment consisted of 20 trials of each of the two problem-types, with the 40 trials presented in a random order. Between each trial, a twelve-second rest was presented to allow for the offset of the hemodynamic response. While it is known that the brain is never really at rest, four 24-s fixation periods were interspersed among the trials to obtain a control baseline measure of brain activation with which to compare the experimental conditions.

fMRI procedure. The study was conducted on a GE 1.5 Tesla scanner used in conjunction with a commercial birdcage, quadrature-drive radio-frequency wholehead coil. Seven oblique-axial images were selected to maximize the coverage of the parietal cortex, the middle frontal gyrus, and the inferior frontal gyrus. The images were collected using a gradient echo, resonant echo planar pulse sequence, with TR $=1500 \mathrm{~ms}$, TE = $50 \mathrm{~ms}$, flip angle $=90^{\circ}$, and a $128 \times 64$ acquisition matrix with a voxel size of $3.125-\mathrm{mm} \times 3.125-\mathrm{mm} \times$ 5-mm with a 1-mm gap. 
Time courses of activation associated with processing the first phrase
Time courses of activation associated with processing the second phrase

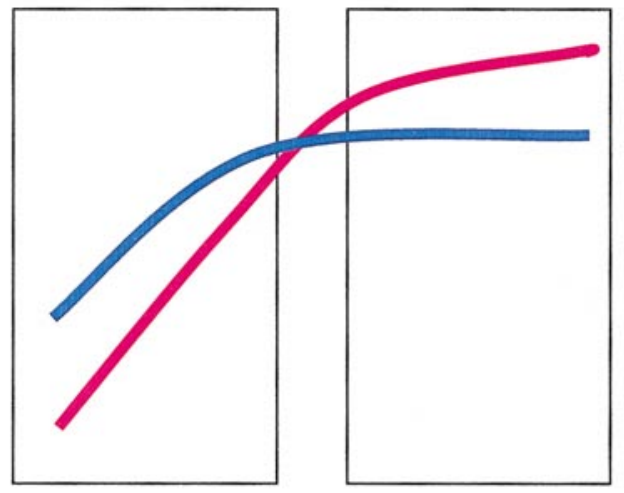

Predicted response for regions affected by the computation of the definite reference.

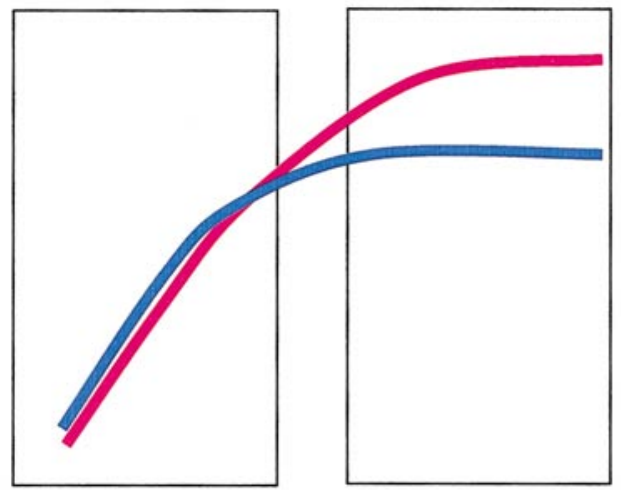

Predicted response for regions affected by the increased working memory load of the late/high load condition due to the postponement of the definite reference computation.

Early/low load
Late/high load

FIG. 1. Two hypothesized time courses for the early/low and late/high load conditions.

I mage preprocessing (including baseline correction, deghosting, mean correction, motion correction, and trend correction) was performed using FIASCO (E ddy et al., 1996; Lazar et al., 2001; further description and tools are available at www.stat.cmu.edu/ fiasco/). The mean of the maximum head motion per participant did not exceed 0.3 voxels. A high-resolution, T1-weighted structural volume scan was obtained for each participant. This volume scan was constructed from 124 3-D SPGR axial images that were collected with $\mathrm{TR}=25$ $\mathrm{ms}, \mathrm{TE}=4 \mathrm{~ms}, 40^{\circ}$ flip-angle, and a $24 \times 18-\mathrm{cm} \mathrm{FOV}$, resulting in $0.9375 \times 0.9375 \times 1.5-\mathrm{mm}$ voxels.

To compare the volume of activation across the two experimental conditions in various regions, anatomical Regions of I nterest (ROIs) were defined individually for each participant. The ROIs were defined using the parcellation scheme of Rademacher and his colleagues (Caviness et al., 1996; Rademacher et al., 1992). This method uses limiting sulci and coronal planes (defined by anatomical landmarks) to segment cortical regions. Because each individuals cortical anatomy is different, the ROIs were drawn on the structural images of each participant to precisely target the anatomical regions of interest. This was done by first computing the mean functional image for each of the functional slices. These mean images were then registered, in parallel alignment with the anterior commissure-posterior commissure (AC-PC) line, to a high-resolution, T1-weighted structural volume scan of each participant. The limiting sulci and other anatomical landmarks were then 


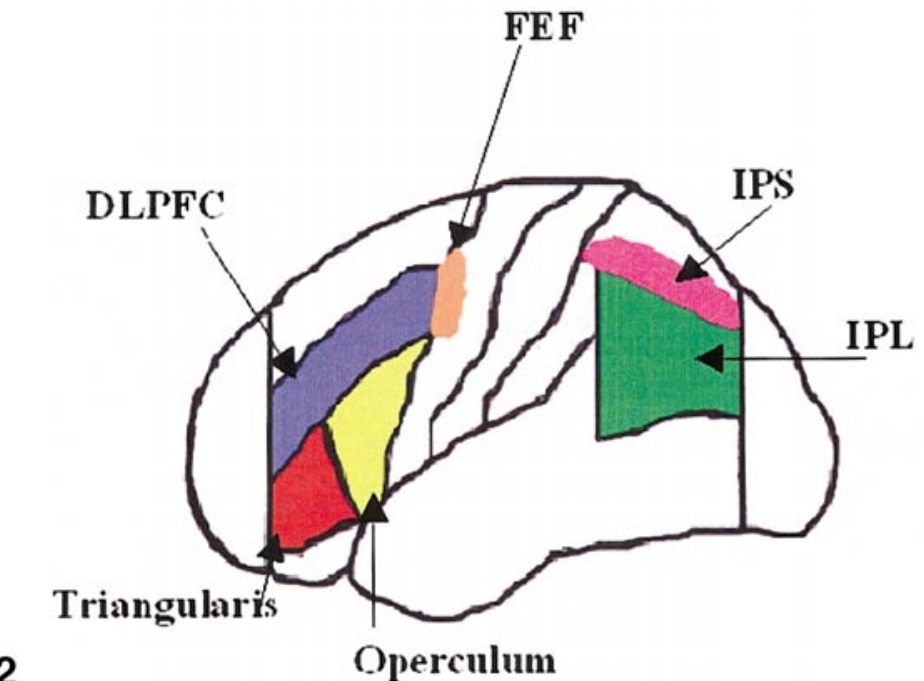

2

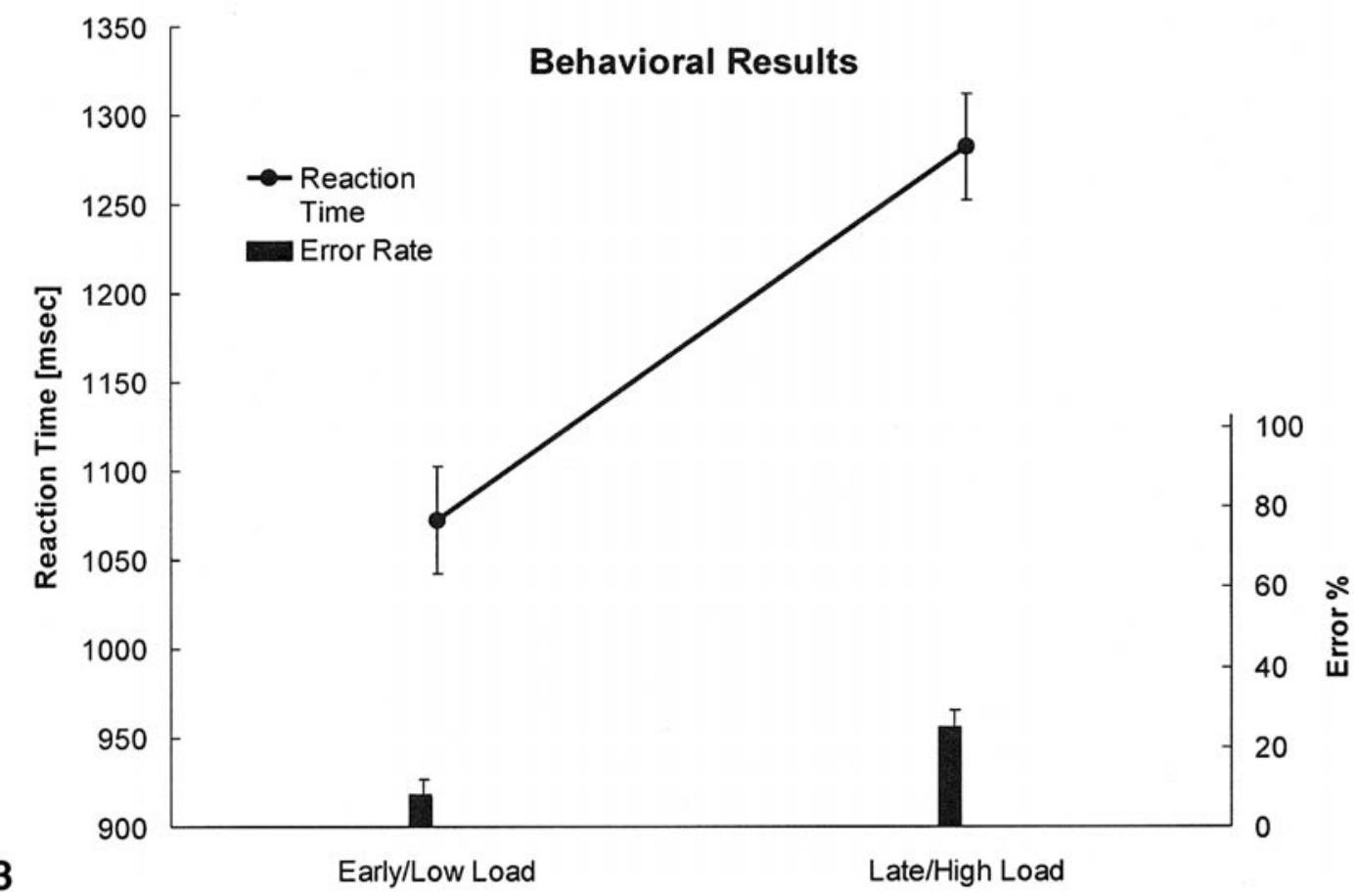

FIG. 2. The regions of interest imposed on a schematic sagittal rendition of the brain. Areas are adaptations from those defined by Rademacher et al. (1992). The regions examined in this study are colored.

FIG. 3. Behavioral results. Error bars represent $95 \%$ confidence intervals based on the pooled Mse from the corresponding ANOVA (Loftus and Mason, 1994).

located by viewing the structural images simultaneously in the three orthogonal planes, and the ROIs were defined by manually tracing the regions onto the axial image of each functional slice (this procedure was completed by the first author). The interrater reliability of this ROI-defining procedure between two trained staff members was previously evaluated for four ROI's in two participants in another study. The reliability measure was obtained by dividing the size of the set of voxels that overlapped between the two raters by the mean of their two set sizes. The resulting eight reli- ability measures were in the $78-91 \%$ range, with a mean of $84 \%$, as high as the reliability reported by the developers of the parcellation scheme.

Because this type of problem solving is the product of large-scale cortical networks (Mesulam, 1990, 1998), the fMRI analyses focused on six relevant ROIs per hemisphere (see Fig. 2) as defined by Caviness et al. (1996). The inferior frontal ROI was divided into two subregions, the operculum ( $F 30$, Brodmann's Areas (BA) 44) and triangularis (F 3t, BA 45). Two parietal ROIs were drawn, the intraparietal sulcus and the 
Dorsolateral Prefrontal Cortex

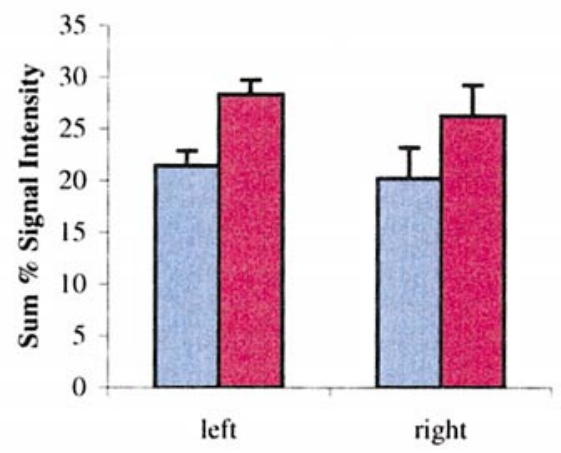

Frontal Triangularis

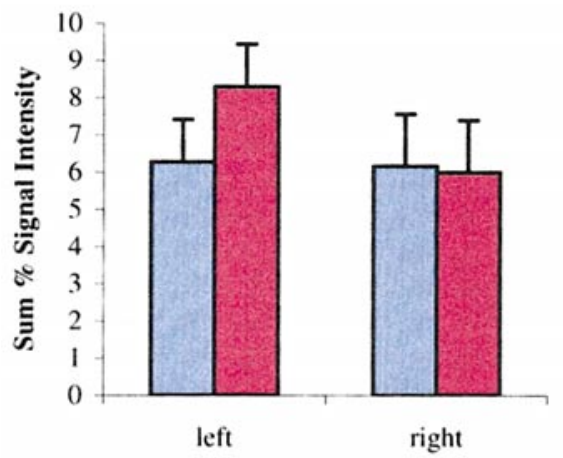

Inferior Parietal Region

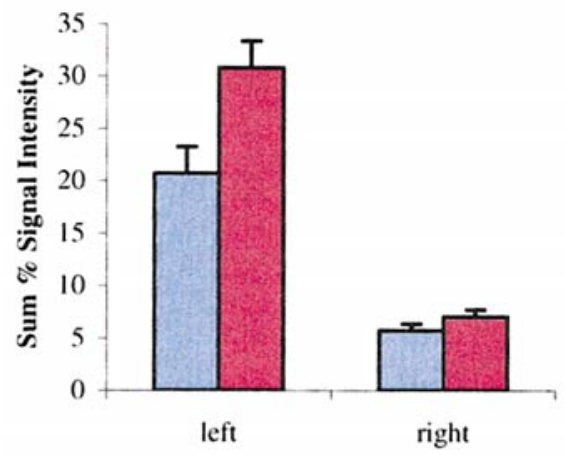

Frontal Operculum

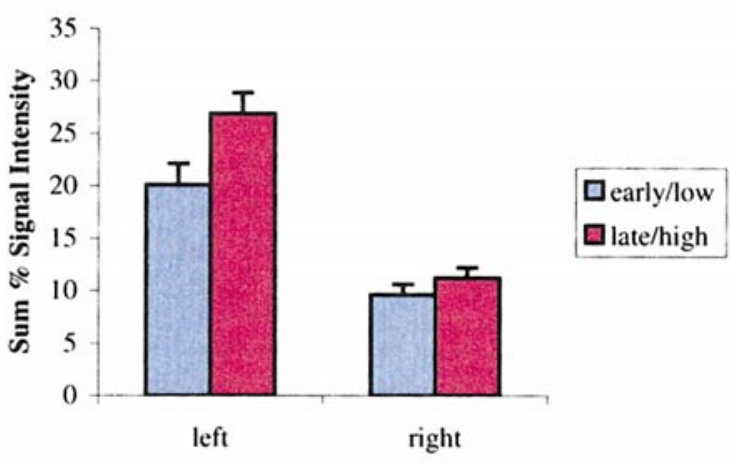

Frontal Eye Fields

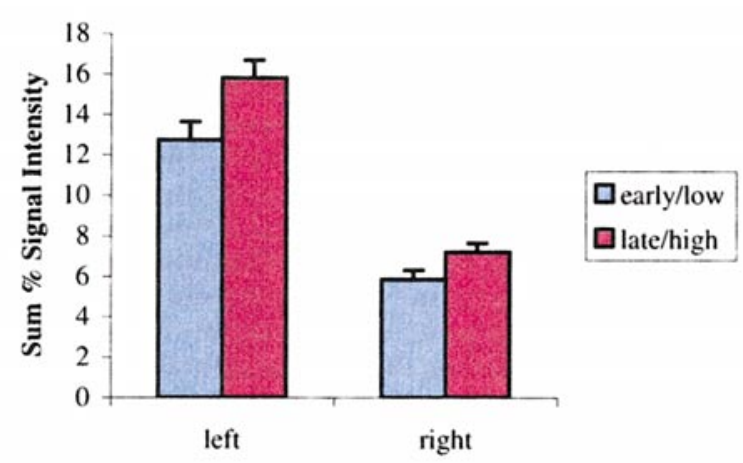

Intraparietal Sulcus

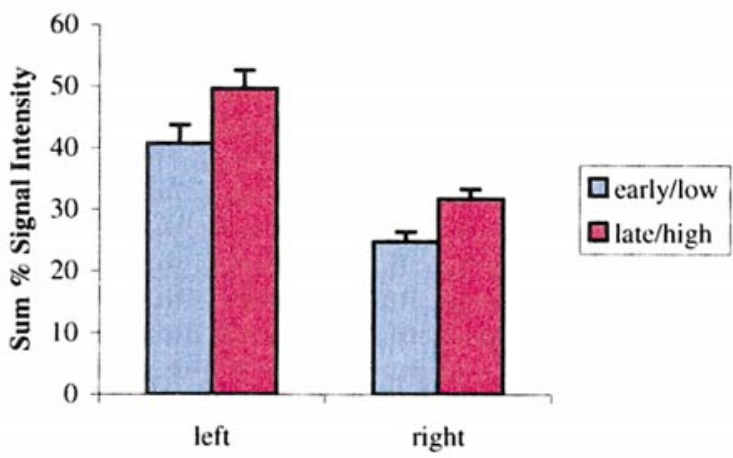

FIG. 4. The sum percentage signal intensity from the ROIs. Error bars represent $95 \%$ confidence intervals based on the pooled Mse from the corresponding ANOVA (Loftus and Mason, 1994).

inferior parietal region. The dorsolateral prefrontal cortex, or DLPFC, ROI corresponds to the middle frontal gyrus (F2; or BA 6, 8, 9, and 46). The frontal eye fields were drawn to include the two posterior-most voxels of $F 2$ in order to exclude it from the DLPFC ROI. Both hemispheres were examined because the right homol ogues of many cortical areas have been shown to be activated by the same type of computational processing as their left counterparts in language comprehension tasks (J ust et al., 1996).
fMRI data analysis. Two steps were taken to insure that the fMRI-measured activation was due to changes in cortical microvascular activity rather than changes in the blood-flow rate of larger vessels. First, the activation maps that were defined by the ROIs corresponded to cortical tissue and not to the spaces normally occupied by cerebrospinal fluid or large blood vessels. The distribution of activation was thus confined to a discrete volume in the image space that did not correspond to the known drainage pattern of large 


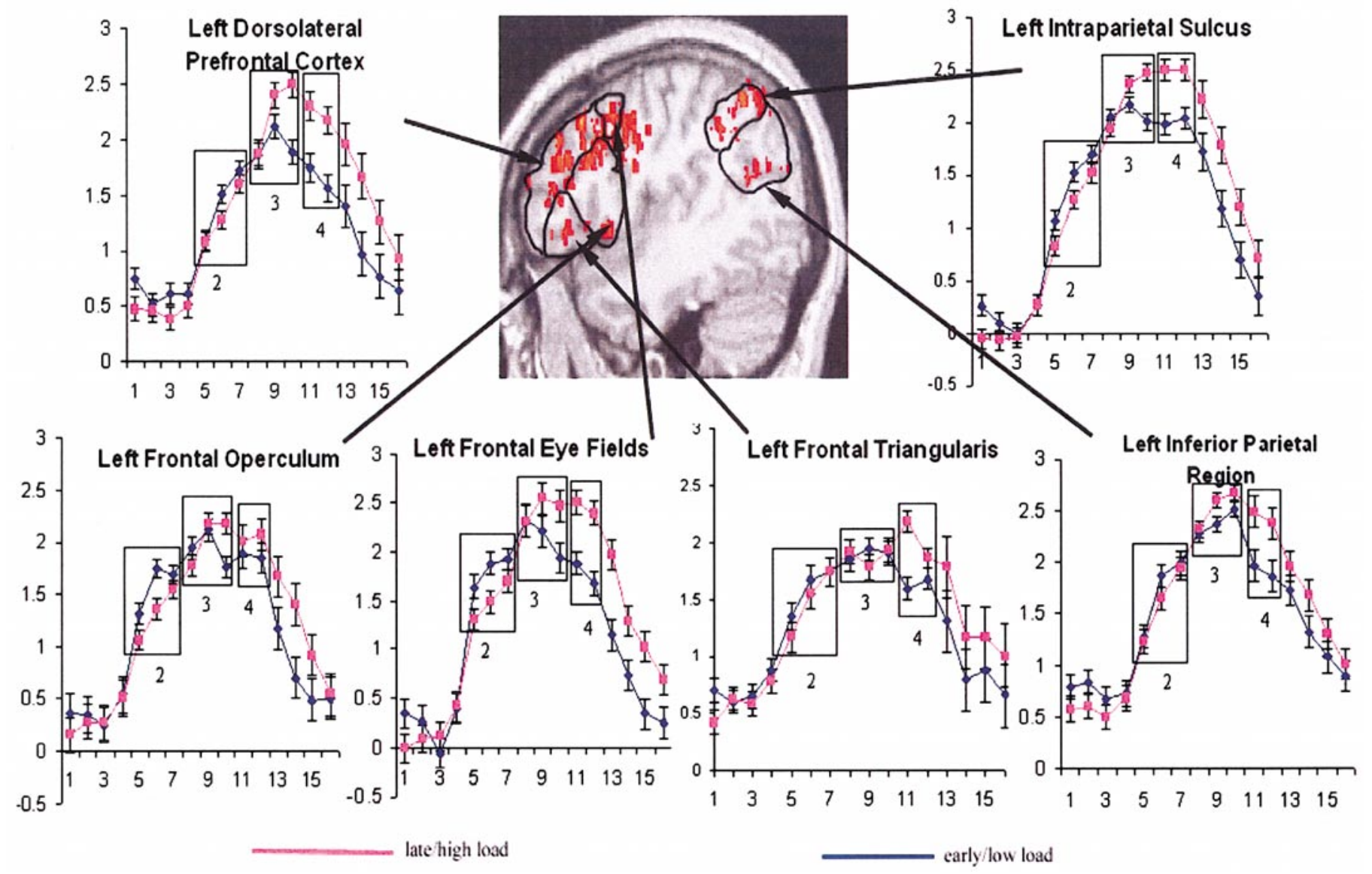

FIG. 5. The Talairach averaged activation from a single sagittal slice along with the time courses from each of the left hemisphere ROIs. Error bars represent 95\% confidence intervals based on the pooled Mse from the corresponding ANOVA (Loftus and Mason, 1994).

veins. Second, to reduce the influence of large blood vessels, any voxel that showed an excessively large percentage change in signal intensity (greater than $6.2 \%$ ) was excluded from the analyses.

As stated in the introduction and depicted in Fig. 1, the principal interest in the current study was the prediction that the time course of the activation in the various regions would differ for the two types of problems, corresponding to the time at which the peak processing demands occurred. Therefore, the $16 \mathrm{im}$ ages (acquired every 1500 ms) obtained during each trial were divided into 5 periods, with an attempt to divide them into psychologically meaningful segments, while taking into account the hemodynamic response function. The underlying neuronal response is delayed and distributed over time by the hemodynamic (BOLD) response. The hemodynamic delay is approximately $6 \mathrm{~s}$, in the sense that that the peak response to a stimulus occurs about six seconds after stimulus onset (Bandettini et al., 1992). Therefore images 1- 4 are label ed as period 1 and correspond to the hemodynamic delay. The next $4.5 \mathrm{~s}$, images 5-7, were labeled period 2 and correspond to the peak activation due to the processing of the first phrase. Note that the first phrase was presented for $4.5 \mathrm{~s}$. The next $4.5 \mathrm{~s}$, images 8-10, were labeled period 3 and correspond to the peak activation due to the processing of the second phrase. Note that the second phrase was presented for $4.5 \mathrm{~s}$. The next 3 s, images 11 and 12, were labeled period 4 and correspond to the peak activation due to the processing of the comprehension probe. Finally, the last $6 \mathrm{~s}$, images 13-16, labeled period 5, correspond to the hemodynamic response offset (see Table 1 ). The analyses focus on periods 2,3 , and 4 where the effects of the cognitive demands are most pronounced. While the effect of each portion of the problem is dispersed throughout the time course due to the smearing function of the hemodynamic response, periods 2,3 , and 4 represent the peak activation due to the processing of the two phrases of the sentences and the probe, respectively. The time course analysis was performed on those voxels that were found to be activated in both experimental conditions.

The fMRI-measured activation was quantified in three additional ways. First, the difference between each voxel's activation in each condition and the base- 

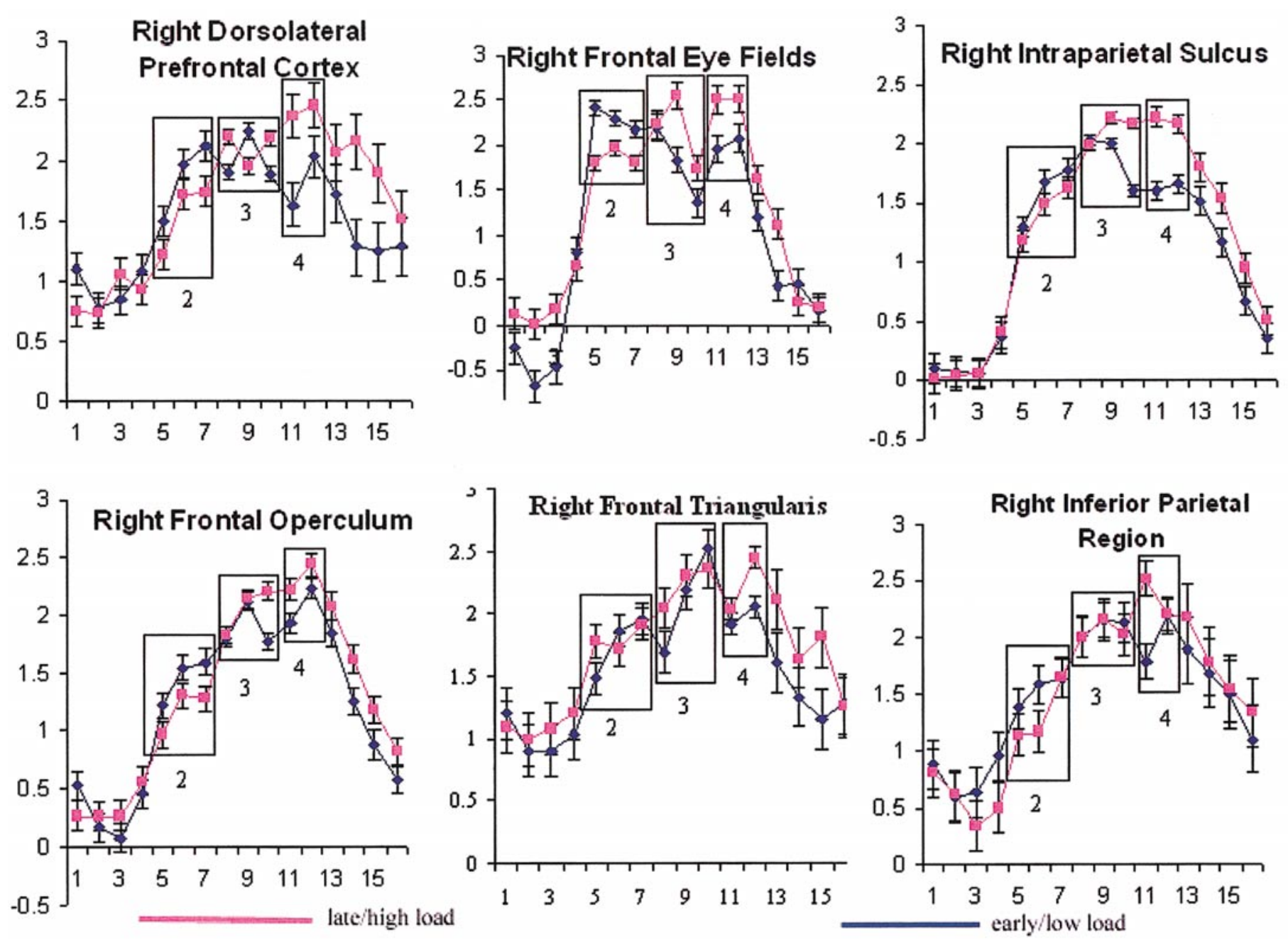

FIG. 6. Time courses from each of the right hemisphere ROIs. Error bars represent $95 \%$ confidence intervals based on the pooled Mse from the corresponding ANOVA (Loftus and Mason, 1994).

line condition was used to construct distributions of $\mathrm{t}$ values within each ROI. Voxels having activation values that exceeded their baseline values (as determined by a t test with $\mathrm{t}>4.5$, which is more conservative than the Bonferroni correction for $P<0.01$ ) were then counted, and the mean number of activated voxels within each ROI was calculated for each condition. The second measure was the mean percent increase in the amplitude of activation relative to the baseline condition for those voxels included in the first measure. The third technique involved comparing the sum of the changes in signal intensity for the set of activated voxels. This was done by adding the percentage change in signal intensity for each voxel activated in a particular condition and comparing this integral measure across conditions.

Functional connectivity. The functional connectivity, a measure of the comodulation or synchronization of two ROIs, was also computed. The rationale

TABLE 1

Time Course Segmentation

\begin{tabular}{|c|c|c|c|c|c|}
\hline & $\begin{array}{c}\text { Hemodynamic } \\
\text { delay }\end{array}$ & $\begin{array}{l}\text { Max. response due } \\
\text { to first phrase }\end{array}$ & $\begin{array}{l}\text { Max. response due } \\
\text { to second phrase }\end{array}$ & $\begin{array}{l}\text { Max. response } \\
\text { due to probe }\end{array}$ & $\begin{array}{l}\text { Hemodynamic } \\
\text { offset }\end{array}$ \\
\hline $\begin{array}{l}\text { Period } \\
\text { Images }\end{array}$ & $\begin{array}{l}\text { Period } 1 \\
1-4\end{array}$ & $\begin{array}{c}\text { Period } 2 \\
5-7\end{array}$ & $\begin{array}{l}\text { Period } 3 \\
8-10\end{array}$ & $\begin{array}{l}\text { Period } 4 \\
11,12\end{array}$ & $\begin{array}{c}\text { Period } 5 \\
13-16\end{array}$ \\
\hline
\end{tabular}




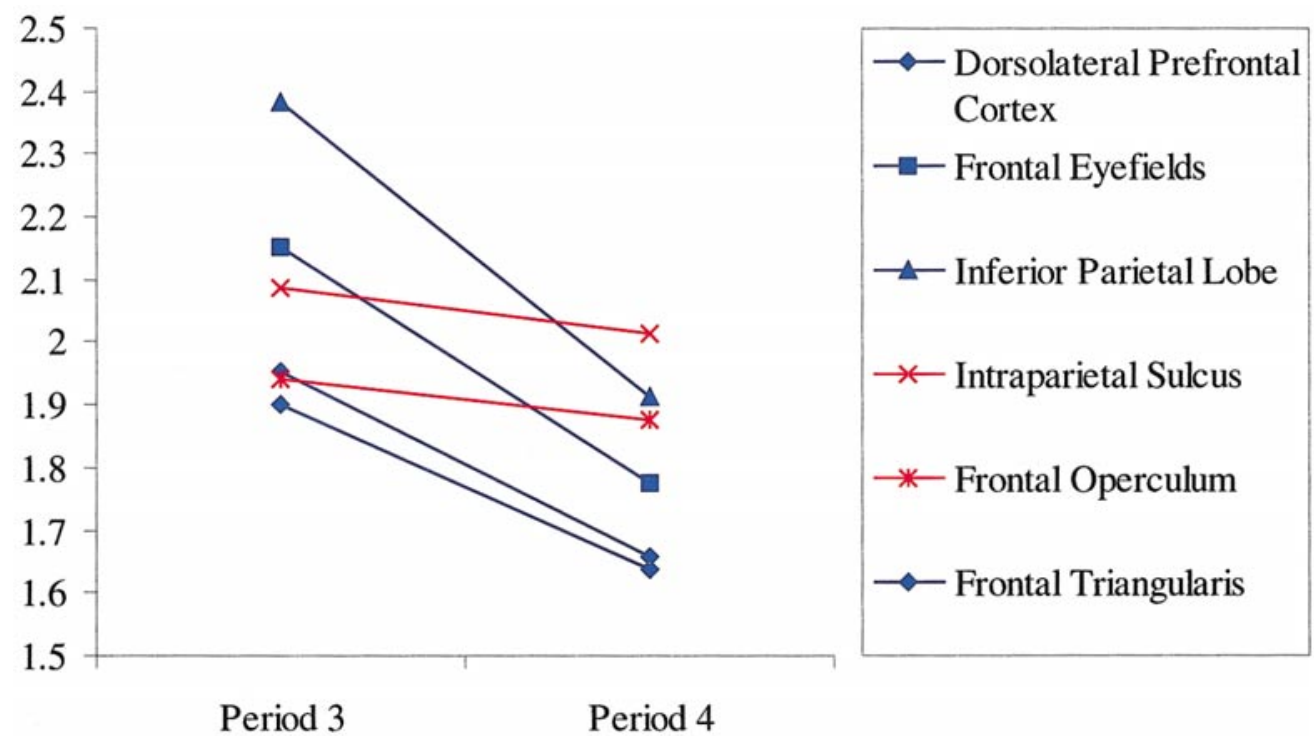

FIG. 7. The average MR amplitude response for periods 3 and 4 for each of the left hemisphere ROI s for the early/low load condition.

behind functional connectivity analysis is that regions that work together have similar temporal response profiles. An example of this approach appears in Diwadkar et al. (2000), where the functional connectivity between frontal and parietal areas increased with cognitive workload in a spatial working memory task. Briefly, the processed data were linearly interpolated in time to correct for the interleaved slice acquisition sequence. A mean time-course of the activated voxels in each ROI was then computed for each of the experimental conditions. This was done separately for each participant and each ROI. The mean correlations (averaged across participants) between the time-courses in pairs of ROls were then computed. In order to determine whether the functional connectivity between regions is modulated by experimental condition, t tests compared the time-course correlations for the 14 participants in the two conditions for various pairs of ROIs.

\section{RESULTS}

\section{Behavioral Measures}

The response times and error data confirmed that the late/high load condition is indeed more difficult than the early/low load condition (see Fig. 3). The response times were on average $210 \mathrm{~ms}$ longer $[\mathrm{F}(1,13)=$ $24.89, \mathrm{P}<0.01$ ], and the error rates were on average $17 \%$ higher for the late/high load compared to the early/low load condition $[F(1,13)=8.98, P<0.01]$.

\section{fMRI Results}

Summary. As expected, the late/high load condition elicited more activation than the early/low load condition in several regions, including the dorsolateral prefrontal cortex (DLPFC), the inferior frontal region, and parietal regions (see Fig. 4). The 6 ROI s per hemisphere were: DLPFC, frontal operculum, frontal triangularis, frontal eye fields, inferior parietal region, and the intraparietal sulcus. An ANOVA examining the effects of condition, ROI, and hemisphere as withinsubject variables revealed that the effect of load significantly affected the overall amount of activation $[F(1,13)=12.04, P<0.005]$. In addition, this task was significantly left lateralized with the effect of condition differentially affecting the left hemispheric regions as shown by the significant condition by laterality interaction $[\mathrm{F}(1,13)=16.07, \mathrm{P}<0.005 ; \mathrm{F}(1,13)=9.59, \mathrm{P}<$ 0.01].

The time course analysis revealed that the early/low load condition generally produced a greater signal change in those images that represent peak activation due to the first phrase while the late/high load condition produced a greater signal change in those images that represent peak activation due to the second phrase and the probe (Fig. 5). A detailed discussion of the activation in specific anatomically-defined cortical regions follows below.

Dorsolateral prefrontal regions. There was significantly more activation related to the processing of the late/high load condition than the early/low load condition $[F(1,13)=5.48, P<0.05]$ (see Fig. 4). The activation was bilateral, with no effect of hemisphere, $\mathrm{F}<1$. Further analysis, however, indicated that only the left DLPFC revealed a significant effect of $\operatorname{Ioad}[F(1,13)=$ $11.46, \mathrm{P}<0.005$ for the left; $\mathrm{F}(1,13)=1.97, \mathrm{P}>0.18$ for the right].

The time course of the activation in left DLPFC 
TABLE 2

Time Course Analysis Statistics

\begin{tabular}{|c|c|c|c|c|c|c|}
\hline \multirow[b]{2}{*}{ ROIs } & \multicolumn{2}{|c|}{ First phrase } & \multicolumn{2}{|c|}{ Second phrase } & \multicolumn{2}{|c|}{ Probe } \\
\hline & $F(1,13)$ & $\begin{array}{l}\text { Signal change } \\
\text { difference }\end{array}$ & $F(1,13)$ & $\begin{array}{l}\text { Signal change } \\
\text { difference }\end{array}$ & $F(1,13)$ & $\begin{array}{l}\text { Signal change } \\
\text { difference }\end{array}$ \\
\hline Left DLPFC & 3.46 & 0.13 & $10.67 *$ & -0.3 & $21.99 * *$ & -0.59 \\
\hline Left operculum & $11.48^{* *}$ & 0.26 & 1.48 & -0.1 & 1.29 & -0.16 \\
\hline Left IPL & 1.27 & 0.11 & $5.79 *$ & -0.15 & $11.21^{* *}$ & -0.52 \\
\hline Left IPS & $9.04 *$ & 0.22 & 7.33* & -0.18 & $22.17^{* *}$ & -0.49 \\
\hline Left triangularis & 1.04 & 0.10 & 0.06 & 0.02 & $17.64^{* *}$ & -0.4 \\
\hline Left FEF & $11.55^{* *}$ & 0.32 & 4.86 & -0.29 & $34.37 * *$ & -0.68 \\
\hline Right DLPFC & $7.05^{*}$ & 0.30 & 3.11 & -0.10 & $8.86^{*}$ & -0.58 \\
\hline Right operculum & $5.72 *$ & 0.27 & 4.49 & -0.17 & 5.2 & -0.25 \\
\hline Right IPL & 1.95 & 0.21 & $<1$ & 0.03 & $5.52 *$ & -0.38 \\
\hline Right IPS & 4.04 & 0.15 & $49.56 * *$ & -0.25 & $56.61^{* *}$ & -0.57 \\
\hline Right triangularis & $<1$ & -0.04 & $<1$ & -0.10 & 3.46 & -0.26 \\
\hline Right FEF & $10.85 *$ & 0.43 & 2.81 & -0.38 & 2.83 & -0.49 \\
\hline
\end{tabular}

$* \mathrm{P} \leq 0.05 ; * * \mathrm{P} \leq 0.005$.

revealed that the activation level was substantially higher for the late/high load problems in period 3 (corresponding to the peak processing of the second phrase), and no such response during period 2 (corresponding to the peak processing of the first phrase) as shown in Fig. 5 and Table 2. This response was pre dicted for regions primarily affected by the increased load effect (Fig. 1B). This is further evidenced by significant load by period interaction in left DLPFC $[F(1,13)=12.63, \mathrm{P}<0.01]$ when comparing periods 2 and 3 for the two problem types.

The time course observed in the right DLPFC re vealed very different characteristics than its left homologue (see Table 2 and Fig. 6). While the region did reveal a significantly greater level of activation during period 2 for the early/low condition there were no differences due to load during period 3 (see Table 2). However, the interaction between load and period when comparing periods 2 and 3 was significant $[F(1,13)=9.47, P<0.05]$.

Frontal opercular regions. The activation within the opercular region of the inferior frontal gyrus was concentrated at the junction of the inferior frontal sulcus and the precentral sulcus (BA 44/6). The activation was significantly modulated by $\operatorname{load}[F(1,13)=4.98$, $\mathrm{P}<0.05]$. In addition, the activation was found to be reliably left lateralized $[\mathrm{F}(1,13)=12.9, \mathrm{P}<0.005]$; furthermore, the left operculum revealed significant effects of load, whereas its right homologue did not $[F(1,13)=5.69, P<0.05 ; F(1,13)=1.3, P>0.2]$.

An analysis comparing periods 2 and 3 in the left operculum revealed a significant interaction between load and period $[\mathrm{F}(1,13)=23.09, \mathrm{P}<0.01]$, primarily due to a larger increase in signal change during period 2 (corresponding to peak processing of the first phrase) for the early/low load condition, the only period that revealed reliable load differences (see Fig. 5 and Table 2).
The right frontal operculum, like its left homologue, revealed a significant interaction between period and condition in the analysis of periods 2 and $3,[F(1,13)=$ 15.33, $\mathrm{P}<0.005$ ] and only period 2 revealed a significant effect of load (see Table 1 and Fig. 6).

Intraparietal sulcal regions. Load significantly modulated the activation in IPS $[F(1,13)=6.77, \mathrm{P}<$ 0.05] (see Fig. 4). In addition, the activation was significantly left lateralized $[F(1,13)=7.9, P<0.05]$. Further analysis revealed that the late/high load condition elicited significantly more activation in both left and right IPS $[\mathrm{F}(1,13)=4.42, \mathrm{P}<0.056 ; \mathrm{F}(1,13)=$ 8.76, $\mathrm{P}<0.05$, respectively].

The time course of the left IPS activation revealed the response predicted for regions primarily involved in the manipulation processes (see Fig. 1A). The left IPS time course revealed a greater signal change due to the first phrase (period 2) for the early/low load condition. The direction of this difference was opposite in period 3 (corresponding to the peak activation due to the second phrase), and continued during the probe processing, as shown in Table 2 and Fig. 5. The left IPS revealed a significant interaction between load and period in the analysis of periods 2 and $3[\mathrm{~F}(1,13)=$ $22.74, \mathrm{P}<0.01]$. This interaction was due to a significantly greater signal change in period 2 (due to the first phrase for the early/low load condition) in contrast to a greater signal change in period 3 (due to the second phrase for the late/high load condition) (see Fig. 5 and Table 2).

Unlike the other activated left hemispheric regions, the left frontal operculum and the left IPS did not show a drop in signal intensity during the early/low load condition between period 3 (corresponding to peak processing of the second phrase) and period 4 (corresponding to peak processing of the probe), as shown in Fig. 7. 
In fact, they were still activated at a high level in period 4.

The right IPS also revealed a significant interaction between period and condition in the analysis of periods 2 and $3[F(1,13)=20.51, P<0.005]$. While there was a trend for the signal intensity to be greater for the early/low load condition during period 2, unlike its left homologue, the right IPS failed to reveal a significant difference here (see Table 2 and Fig. 6).

Frontal triangular regions. The frontal triangularis failed to reveal effects of load, $\mathrm{F}<1$. In addition, the activation within this region was found to be bilateral as indicated by the lack of a significant effect of laterality $[\mathrm{F}(1,13)=1.39, \mathrm{P}>0.2]$ (see Fig. 4).

In the left triangularis, unlike the other regions, there was no significant interaction between load and periods 2 and $3, F<1$. Although periods 2 and 3 do not reveal significant differences in signal change as a function of load, as shown in Table 2, period 4, corresponding to peak activation related to the probe, does (see Fig. 5 and Table 2).

The time course observed in the right frontal triangularis also failed to reveal a significant interaction between period and condition, $\mathrm{F}<1$. In addition, there were no reliable differences between early/low and late/high load condition in any of the three critical periods (see Table 2 and Fig. 6).

Inferior parietal regions. The activation in the inferior parietal region (IPL) was significantly modulated by task difficulty $[\mathrm{F}(1,13)=7.86, \mathrm{P}<0.05]$. In addition, while the activation was found to be left lateralized $[F(1,13)=16.14, P<0.005]$ the effect of difficulty differentially affected the left IPL as indicated by the significant interaction between difficulty and laterality $[\mathrm{F}(1,13)=6.93, \mathrm{P}<0.05]$. Whereas the left IPL revealed significant effects of task difficulty, its right homologue did not $[\mathrm{F}(1,13)=7.84, \mathrm{P}<0.05$; $F(1,13)=2.09, P>0.17]$ (see Fig. 4).

The left inferior parietal region, unlike the left triangularis, revealed a significant interaction between load and period in the analysis of periods 2 and 3 $[F(1,13)=7.58, P<0.02]$ primarily due to an increase in signal change in those images that correspond to the peak activation of the second phrase for the late/high load condition (see Fig. 5 and Table 2).

The time course observed in the right inferior parietal region, unlike its left homologue, failed to show a significant interaction between period and condition when comparing periods 2 and $3, F<1$. The only period that revealed significant differences due to load was period 4, corresponding to the peak activation associated with the processing of the probe (see Table 2 and Fig. 6).

Frontal eye field ROIs. The activation elicited in the frontal eye fields was found to be significantly modulated by load $[\mathrm{F}(1,13)=8.02, \mathrm{P}<0.05]$. In addi- tion, the activation was found to be left lateralized $[F(1,13)=5.97, P<0.05]$. While there was a trend for the late/high load condition to engender more activation than the early/low load condition, it was only reliable in the left FEF $[F(1,13)=5.70, P<0.05$ in left; $F(1,13)=4.14, P>0.06$ in right] (see Fig. 4). The time course of the activation in the left frontal eyefields was similar to that of the left IPS.

The interaction between periods 2 and 3 and load was significant $[F(1,13)=17.91, P<0.005]$ and the early/low load condition revealed significantly more activation than the late/high load condition in period 2 (corresponding to the peak activation associated with the processing of the first phrase (see Table 2). Direction of the difference reverses during period 3, corresponding to peak activation due to the second phrase.

The right frontal eyefields, on the other hand, failed to reveal a significant interaction between load and periods 2 and $3[F(1,13)=6.72, P>0.08]$. While the period corresponding to the peak activation due to the first phrase revealed a significant difference due to load, as shown in Table 2, the other two relevant periods did not (see Tables 2 and 3 and Fig. 6).

Functional connectivity. The functional connectivity between pairs of ROIs was assessed. An ANOVA with load as the independent variable (early/low load versus late/high load) was computed for each ROI pair. For any pair of ROIs, the analysis included only those participants whose correlation was greater than 0.4.

The functional connectivity analysis appears to reveal two networks. The functional connectivity between the left DLPFC and several regions was significantly modulated by task difficulty [the frontal operculum, $F(1,8)=7.89, P<0.05$; the left IPS, $F(1,12)=6.67, P<0.05$; the left IPL, $F(1,7)=12.67$, $\mathrm{P}<0.05$; and the right IPS, $\mathrm{F}(1,8)=10.23, \mathrm{P}<0.05$ ]. In addition, the connectivity between the left intraparietal sulcus and left opercularis was significantly higher in the late/high load condition, $F(1,11)=9.75$, $P<0.05$.

\section{DISCUSSIO N}

The new result obtained here demonstrates the measurement of the differential dynamic time course of cortical network components as they adapt to the computational load that is imposed by the information processing at various points in time. More specifically, the results suggest that increasing the working memory load by manipulating the timing of a particular computation affects a network of regions including both prefrontal and posterior cortex, bilaterally. These regions included the dorsolateral prefrontal cortex, the inferior frontal gyrus, and the parietal lobe.

One of the interesting outcomes highlights the possible differences in the contributions of the left and 
right dorsolateral prefrontal cortex during this verbal reasoning task. The traditional view is that the right hemisphere, in general, is primarily involved in spatial processing, and specifically, the right DLPFC has been implicated in spatial working memory. However, re cent working memory results suggest that the manipulation of the contents of working memory elicits bilateral activation of DLPFC, with a right side preference, regardless of whether the stimuli are spatial or nonspatial (D'E sposito et al., 1999). In addition, the right prefrontal cortex has been shown to exhibit significantly more activation during the maintenance of integrated information (when letters to be remembered were displayed in the locations to be remembered) than during the maintenance of unintegrated information (when the letters to be remembered were displayed centrally and separately from the locations to be re membered) (Prabhakaran et al., 2000). This result held despite the fact that the behavioral measures indicated that the integrated condition was easier than the unintegrated condition. Although the authors interpreted their findings in terms of right DLPFC performing some integration of information, an alternative interpretation is that strategic planning is necessary to integrate and maintain the information. In the current study, the activation within the right DLPFC failed to show condition effects in either the volume of activation, time course, or the functional connectivity. Our result is compatible with right DLPFC playing a strategic planning role, which was necessary in both conditions.

The left DLPFC revealed a different pattern of activation, suggesting its involvement in the cognitive control processes necessary to provide top-down support. This interpretation is consistent with the reliable load effects observed in the volume of activation, time course and the functional connectivity of the left DLPFC. Support for the idea that the left DLPFC is involved in cognitive control has been reported previously. In a single trial fMRI study of the Stroop task, activation within the left DLPFC was found to be modulated only during the condition when subjects are naming the ink col or but not during the condition when subjects are reading the word. This finding is consistent with the role of the left DLPFC in representing and maintaining task demands needed for top-down control (MacDonald et al., 2000).

When participants are solving the late/high load problems in the current task, they may have to buffer the first phrase until after they have processed the second phrase, and only then they can compute the definite reference of the initial phrase and incorporate the information onto the sentence representation. The management of the buffering of the verbal information and its subsequent retrieval and computation of its definite reference in the context of the second phrase appears to tax left DLPFC. Moreover, the type of func- tionality that we ascribe to left DLPFC is similar to the manipulation and coordination of several processes that have been associated with the left DLPFC in other studies (Postle and D'E sposito, 1999; D'E sposito et al., 1999; Owen et al., 1998; Petrides, 1998, 1995; Cohen et al., 1997).

The left opercularis and left IPS appear to work closely together in this task, as indicated by the synchronization of their time courses, their MR amplitude response, and their functional connectivity. The left opercularis and left intraparietal sulcus both show reliably greater signal increases during the first phrase for the early/low load condition compared to the late/ high load condition. They also both show a sustained level of activation during the probe condition compared to other left hemisphere regions (see Fig. 6). In addition, the functional connectivity between the two regions was significantly higher in the late/high load condition further supporting the interpretation that these two regions collaborate. Similar activation has been found during previous studies of the Tower of London task, and the activation within these areas was correlated with the number of moves that have to be planned (Baker et al., 1996; Dagher et al., 1999), suggesting that the frontal operculum may be involved in goal management in the current study. In addition, in a recent study comparing sentences that evoke a mental image and sentences that only relay factual knowledge, the activation within the left operculum and the intraparietal sulcal region was modulated by imagery (Carpenter et al., 2001).

The maintenance of verbal information has been thought to involve the left inferior frontal gyrus and the left inferior parietal lobe. The activation in the left frontal triangularis was found in the inferior portion of the inferior frontal gyrus, in and around the ascending ramus. Several studies have found that the pars triangularis, along with the inferior parietal lobe, is related to the maintenance of information in a phonological form (Petrides, 1995). In the current study both the left triangularis and the left inferior parietal lobe revealed significant effects of load, but fairly late in the time course, specifically during the probe. The load demand during the late/high load condition arises due to the order of the two phrases; specifically, the referent of the first phrase, "my favorite month," cannot be calculated immediately because it presupposes the identity of "the month after April," which is the unknown target or new information. Consequently, this first phrase must be retained while the referent of the second phrase is computed. This second phrase constitutes the given information. Earlier comprehension studies indicated that processing is slower when the given information follows rather than precedes the new information (Haviland and Clark, 1974; Clark, 1977). Furthermore, it has been suggested that the reason for the slower processing is due to a reordering of the 
constituents of the sentence (Carpenter and J ust, 1977). The late affect of load in the left triangularis, left parietal region and left DLPFC are all consistent with the hypothesis that participants rearrange the contents of working memory in order to make the answer-formulation process easier.

Further support was found in the functional connectivity results, with the functional connectivity between left DLPFC and the left inferior parietal lobe significantly greater for the late/high load condition compared to the early/low load condition. If the verbal information being buffered within the inferior parietal region needs to be manipulated during the late/high load condition, then one would expect the collaboration/coordination between the left DLPFC and the inferior parietal region to be greater in the high load than in the low load condition, which was indeed observed.

The task that was used in the current study varies the load and its timing (the location of the load in the problem) simultaneously, a characteristic of this particular problem, which has been the object of considerable previous study (e.g., Casey, 1993). However, it is interesting to consider the possible results of future research in which the effects of these two variables are studied separately. Other studies performed in our lab have investigated the effect of a load manipulation al one without a simultaneous manipulation in the timing of events (Mason et al., submitted). In that study a difference in the magnitude of signal change was observed, but no time course differences analogous to those reported above were found in the time course of the fMRI activation associated with the comprehension of syntactically ambiguous vs unambiguous sentences. It should also be possible to develop new tasks that vary the size of the load in two locations (timings) orthogonally, an interesting issue for future research.

To summarize, the results presented here suggest that increasing the verbal working memory load by manipulating the timing of the required mental calculations affected the timing of the activation in a network of regions, including the dorsolateral prefrontal cortex, the inferior frontal gyrus, and the parietal lobe. The execution of the processes appears to be highly collaborative among the areas. This is particularly true for the frontal operculum and the intraparietal sulcus as well as the dorsolateral prefrontal cortex and the inferior parietal region. The results also suggest that the frontal operculum and the IPS together carry out processes necessary to perform calculations while the left DLPFC, frontal triangularis and the inferior parietal region together perform processes required to buffer and subsequently compute the definite reference of the initial noun phrase during the high-load problems.

It is a truism to say that cortical areas operate as a network, but it is a much greater challenge to deter- mine what each component of the network is doing and to determine how collaboratively or autonomously each component is operating. In fact, the challenge is to determine the workings of a complex system, in the technical sense of the term. This study illustrates the use of a particular approach, time course analysis of $\mathrm{fMRI}$ activation to perform such an analysis. The relation of each component's activation time course in relation to the problem's computational demands provides valuable clues to the components' functions. Equally valuable is the information about the synchronization between particular pairs or triplets of areas, which are suggestive of collaborative subnetworks. As fMRI technology progresses, finer grain temporal analysis should be possible, even with conventional BOLD signals. This improved temporal resolution may allow for a better understanding of not only the individual components of complex neurocognitive systems but also their collaboration.

\section{ACKNOWLEDGMENTS}

This work was supported in part by the National Institute of Mental Health Grant MH-29617 and Senior Scientist Awards MH00661 and $\mathrm{MH}-00662$.

\section{REFERENCES}

1. Awh, E., J onides, J., Smith, E. E., Schumacher, E. H., Koeppe, R. A., and Katz, S. 1996. Dissociation of storage and rehearsal in verbal working memory: Evidence from positron emission tomography. Psychol. Sci. 7: 25.

2. Baker, S., et al. 1996. Neural systems engaged by planning: A PET study of the Tower of London task. Neuropsychologia 34: 515.

3. Bandettini, P. A., Wong, E. C., Hinks, R. S., Tokofsky, R. S., and Hyde, J. S. 1992. Time course EPI of human brain function during task activation. Magn. Reson. Med. 25: 390.

4. Carpenter, P. A., J ust, M. A., and McEleney, A. Mental imagery during sentence comprehension. In preparation.

5. Casey, P. J . 1993. "That man's father is my father's son": The role of structure, strategy, and working memory in solving convoluted verbal problems. Memory Cogn. 21: 506.

6. Caviness, V. S. J r., Meyer, J ., Makris, N., and Kennedy, D. N. 1996. MRI-based topographic parcellation of human neocortex: An anatomically specified method with estimate of reliability. J . Cogn. Neurosci. 8: 566.

7. Clark, H. 1977. Inferences in Comprehension. In Basic Processes in Reading: Perception and Comprehension (LaBerge, D., and Samuels, S. J ., Eds.), pp. 243-264. Ablex, NJ .

8. Clark, H., and Haviland, S. 1977. In Discourse Production and Comprehension (R. Freedle, Ed.) Ablex, NJ .

9. Cohen, J ., et al. 1997. Temporal dynamics of brain activation during a working memory task. Nature 386: 604.

10. D'Esposito, M., Postle, B., Ballard, D., and Lease, J . 1999. Maintenance versus manipulation of information held in working memory: An event-related fMRI study. Brain Cogn. 41: 66.

11. Dagher, A., Owen, A., Boecker, H., and Brooks, D. 1999. Mapping the network for planning: A correlational PET activation study with the Tower of London. Brain 122: 1973. 
12. Dehaene S., Spelke, E., Pinel, P., Stanesce, R., and Tsivkin, S. 1999. Sources of Mathematical thinking: Behavioral and brainimaging evidence. Science 284: 970.

13. Diwadkar, V. A., Carpenter, P. A., and J ust, M. A. 2000. Collaborative Activity between Parietal and Dorsolateral prefrontal cortex in dynamic spatial working memory revealed by $\mathrm{fMRI}$. Neuroimage 12: 85.

14. Eddy, W., Fitzgerald, M., Genovese, C., Mockus, A., and Noll, D. 1996. Functional imaging analysis software-Computational olio. In Proceedings in Computational Statistics, pp. 39-49. Physica-Verlag, Heidel berg.

15. J onides, J ., Schumacher, E. H., Smith, E. E., Koeppe, R. A., Awh, E., Reuter-Lorenz, P. A., Marshuetz, C., and Willis, C. R. 1998. The role of parietal cortex in verbal working memory. J . Neurosci. 18: 5026-5034.

16. Lazar, N. A., Eddy, W. F., Genovese, C. R., and Welling, J . S. 2001. Statistical Issues in fMRI for Brain Imaging. Int. Stat. Rev.

17. Mason, R., J ust, M., Carpenter, P., and Keller, T. Ambiguity in the brain: What brain imaging reveals about the processing of syntactically ambiguous sentences. Submitted.

18. McDonald, A. W., Cohen, J. D., Stenger, V. A., and Carter, C. S. 2000. Dissociating the role of the dorsolateral prefrontal and anterior cingulate cortex in cognitive control. Science 288: 1835.
19. Mesulam, M-M. 1990. Large-scale neurocognitive networks and distributed processing for attention, language and memory. Ann. Neurol. 28: 597.

20. Mesulam, M-M. 1998. From sensation to cognition. Brain 121: 1013.

21. Owen, A., et al. 1998. Functional organization of spatial and nonspatial working memory processing within the human lateral frontal cortex. Proc. Natl. Acad. Sci. 95: 7721.

22. Prabhakaran, V., Narayanan, K., Zhao, Z., and Gabrieli, J . D. E. 2000. Integration of diverse information in working memory within the frontal lobe. Nature Neurosci. 3: 85.

23. Petrides, M. 1995. Functional organization of the human frontal cortex for mnemonic processing: Evidence from neuroimaging studies. Ann. N.Y. Acad. Sci. 769: 85.

24. Petrides, M. 1998. Specialized systems for the processing of mnemonic information within the primate frontal cortex. In The Prefrontal Cortex: Executive and Cognitive Functions (A. C. Roberts, T. W. Robbins, and L. Weiskrantz, Eds.), pp. 103-116. Oxford Univ. Press, Oxford.

25. Postle, B., and D'Esposito, M. 1999. "What"-then- "where" in visual working memory: An event-related fMRI study. J . Cogn. Neurosci. 11: 585.

26. Rademacher, J ., Galaburda, A. M., Kennedy, D. N., Flilipek, P. A., and Caviness, V. S. 1992. Human cerebral cortex: Localization, parcellation, and morphometry with magnetic resonance imaging. J . Cogn. Neurosci. 4: 352. 